\title{
Expanding the phenotype of MOG-antibody associated disease (MOGAD): \\ half a century of epilepsy and relapsing optic neuritis
}

Kleerekooper, I. (MD) $)^{1,2}$, Trip, S.A. (FRCP, MD, PhD) ${ }^{3}$, Plant, G.T. (FRCP, FRCOphth, MD, FEAN) ${ }^{3}$, Petzold, A. (FRCP, MD, PhD) $)^{1,2,4^{*}}$

\section{Affiliations}

${ }^{1}$ Moorfields Eye Hospital City Road, London, UK.

2 National Hospital for Neurology and Neurosurgery, UCLH, Queen Square, London, UK.

${ }^{3}$ UCL Institute of Neurology, London, UK.

${ }^{4}$ Dutch Expertise Centre Neuro-ophthalmology, Amsterdam UMC, The Netherlands

* Corresponding author: Axel Petzold, The National Hospital for Neurology and Neurosurgery, Queen Square, London, WC1N 3BG, United Kingdom. [E]: a.petzold@ucl.ac.uk

Key words: optic neuritis, epilepsy, synchronised paroxysmal symptoms, autoimmune, steroid responsive 
Autoimmune disease has opened a field for discovery of new phenotypes in clinical neurology. The unravelling of the autoimmune pathophysiology has led to successful novel treatment strategies including long-term immune suppression [1]. In myelin oligodendrocyte glycoprotein antibody associated disease (MOGAD) damage to the central nervous system is attack related [2]. The clinical phenotype of MOGAD is heterogenous and expanding. Here we a illustrate a new phenomenon in a MOGAD seropositive patient whom we have followed-up for 48 years: the synchronisation of relapsing, steroid responsive epilepsy and optic neuritis (ON). The longterm follow-up with repeated imaging, electrodiagnostic and laboratory tests of blood and cerebrospinal fluid permits to exclude with confidence that this phenotype is due to the more commonly described association of seizure activity with ON in acute encephalitis [3].

The 69 year old woman with relapsing ON reports these attacks to be often preceded by clusters of seizures. 48 years earlier she was diagnosed with epilepsy (1973, Figure 1A). The pattern of epilepsy evolved and presently she has strictly nocturnal, complex partial seizures of left frontal lobe onset, with occasional tongue biting and/or brief jerking of all limbs. The following day she is fatigued, but she had never been encephalopathic. She also suffers from periodic limb movements during sleep which rouse her with sleep paralysis. Repeated electroencephalograms and polysomnography demonstrated consistently a left temporal epileptogenic zone. Repeated CT and MR brain imaging was normal. Her epilepsy was treatment refractory to carbamazepine in spite of being compliant and repeated blood levels being in the therapeutic range. Her seizure frequency remained at three to four times per month for almost 20 years. Seizure frequency reduced, 
however to 3-5 per year up to the present time after treatment with corticosteroids was started because of left relapsing ON (1991, case \#14 in [4]). Serial MRI scans revealed isolated enhancement of the left optic nerve in attacks. Brain imaging was normal and there was no evidence for a myelopathy. The ON was corticosteroid responsive and dependent (threshold at prednisolone 5.5mg once daily, 1993), which led to a clinical diagnosis of chronic relapsing inflammatory optic neuropathy (CRION) [4]. Her maintenance therapy has now been combined prednisolone and mycophenolate for over ten years. It was not until the relevance of MOG-antibodies was rediscovered that evidence for the presumed autoimmune pathology underlying the clinical diagnosis of CRION could be demonstrated [2]. She tested MOG seropositive twice (Figure 1A, 2015, 2016). At the time, the novel reported association of MOGAD with epilepsy in acute encephalitis was considered and excluded. Panel analysis came back normal for antibodies targeting aquaporin-4, N-methyl-D-aspartate (NMDA) and voltage-gated potassium channels (CASPR-2 and LGI-1) as well as ANA, ANCA and paraneoplastic antibodies. Her cerebrospinal fluid examination was normal including absence of evidence for intrathecal IgG synthesis on two occasions (1991, 1996).

The patient highlighted that her ON relapses are always preceded by an increase of seizure activity. Clinically our advice over the past 29 years had been to increase her corticosteroid dosage immediately when noticing first ON symptoms, to protect vision [5]. Remarkably, this typically also arrested seizure activity. Finally, she started to keep a detailed diary of her seizure and ON attacks (2015-2018). Over this four year observation period it became evident that episodes of $\mathrm{ON}$ were preceded by seizures 1-2 days prior for three out of five events (Figure 1A). Importantly, the increased corticosteroid dose (10$20 \mathrm{mg}$ once daily) always stopped the seizures and on three occasions seizures reoccurred upon oral corticosteroid taper. 
At her last clinical assessment (04-DEC-2019) she reported left red desaturation and had a left relative afferent pupillary deficit. Visual acuities were well preserved at 0.00 logMAR, potentially attributable to the consistent high-dose corticosteroid treatment self-initiated immediately at $\mathrm{ON}$ relapse [5]. There was bilateral optic disc pallor. Optical coherence tomography of the macula showed severe atrophy of the ganglion cell and inner plexiform layers which has remained stable over the past six years (Figure 1B). The inner nuclear layer was not thickened and there was no evidence for retrograde maculopathy (microcystic macular oedema). She had not suffered from COVID-19 when we last spoke to her in August 2020.

Seizures can occur in MOGAD. The seizure prevalence in MOGAD is $5-21 \%$, compared to 0.4-1\% in AQP4-antibody seropositive NMOSD [3]. Seizure phenotypes in MOGAD are diverse, although most have a focal onset, are monophasic and MRI shows isolated or disseminated encephalitis. Several cases of concurrent ON and epilepsy in MOGAD have been reported, but all had a mono- or biphasic disease course with inflammatory lesions on MRI [3]. Some patients have normal imaging at initial presentation but develop more extensive clinical and radiological disease shortly after. Here, we describe a long-lasting syndrome of corticosteroid responsive epilepsy frequently synchronised with relapsing ON in adult MOGAD. This suggests that both conditions may be immune mediated and potentially be summarised as synchronised steroid responsive epilepsy with relapsing ON (SERON). Although our patient was seronegative for antibodies associated with autoimmune epilepsy, we cannot exclude the possibility there is a different, unidentified auto-antibody. Importantly, this case highlights that MOG antibodies may be present in presumed auto-immune mediated, antibody negative epilepsy with normal brain imaging. 
In conclusion, early recognition of SERON requires investigation for MOG-antibody and prompt initiation of corticosteroid treatment. The likely critical interval for starting corticosteroids is within less than 48 hours of symptoms onset [5]. This should be followed by a slow oral taper of corticosteroids to test for corticosteroid dependence [5]. Epileptic activity in patients with MOGAD may prove to show more commonly a corticosteroidresponsive phenotype, as in this case.

It has taken us half a century to realise that in our patient the synchronisation of paroxysmal attacks affecting different parts of the central nervous system were interdependent and that both disorders responded to immune suppression. It is therefore intriguing to speculate if a more careful revision of the sometimes poorly understood plethora of paroxysmal symptoms in our patients may indeed reveal other novel phenotypes. We propose that the recognition the synchronous appearance of such paroxysmal symptoms provides an important clinical clue. Testing for auto-antibodies and a corticosteroid trial is recommended in such cases. 


\section{Acknowledgements}

This study was approved by Moorfields Eye Hospital R\&D (study number

CaRS_PETA_01) and the patient has given written informed consent for publication. Iris Kleerekooper thanks the ECTRIMS for their support through the Postdoctoral International Research Fellowship. S Anand Trip receives support from the NIHR Biomedical Research Centre at University College London Hospitals NHS Foundation Trust and University College London. AP was supported by the National Institute for Health Research (NIHR) Biomedical Research Centre based at Moorfields Eye Hospital NHS Foundation Trust and UCL Institute of Ophthalmology. The views expressed are those of the author(s) and not necessarily those of the NHS, the NIHR or the Department of Health. We thank Professor John Duncan for his shared clinical care of the described patient and helpful discussions on the case. We apologise to those authors whom we were not able to cite because of space limitations of the letter format. Additional references can be requested from the authors.

\section{Conflict of interest and source of funding}

IK reports no disclosures. SAT and PGT report no relevant disclosures. AP is a member of the steering committee for the OCTiMS study (Novartis) and ARI network (Zeiss), no consulting fees. Performed OCT QC for the Passos study (Novartis), received consulting fees. Received speaker fees from Heidelberg Engineering. This study was not funded.

\section{Contributionship Statement}

Study design: AP, Laboratory work: AP, Data collection: IK, AP, Data analyses and interpretation: IK, AT, GTP, AP, Manuscript writing: AP, IK, Manuscript revision: all coauthors. 
A

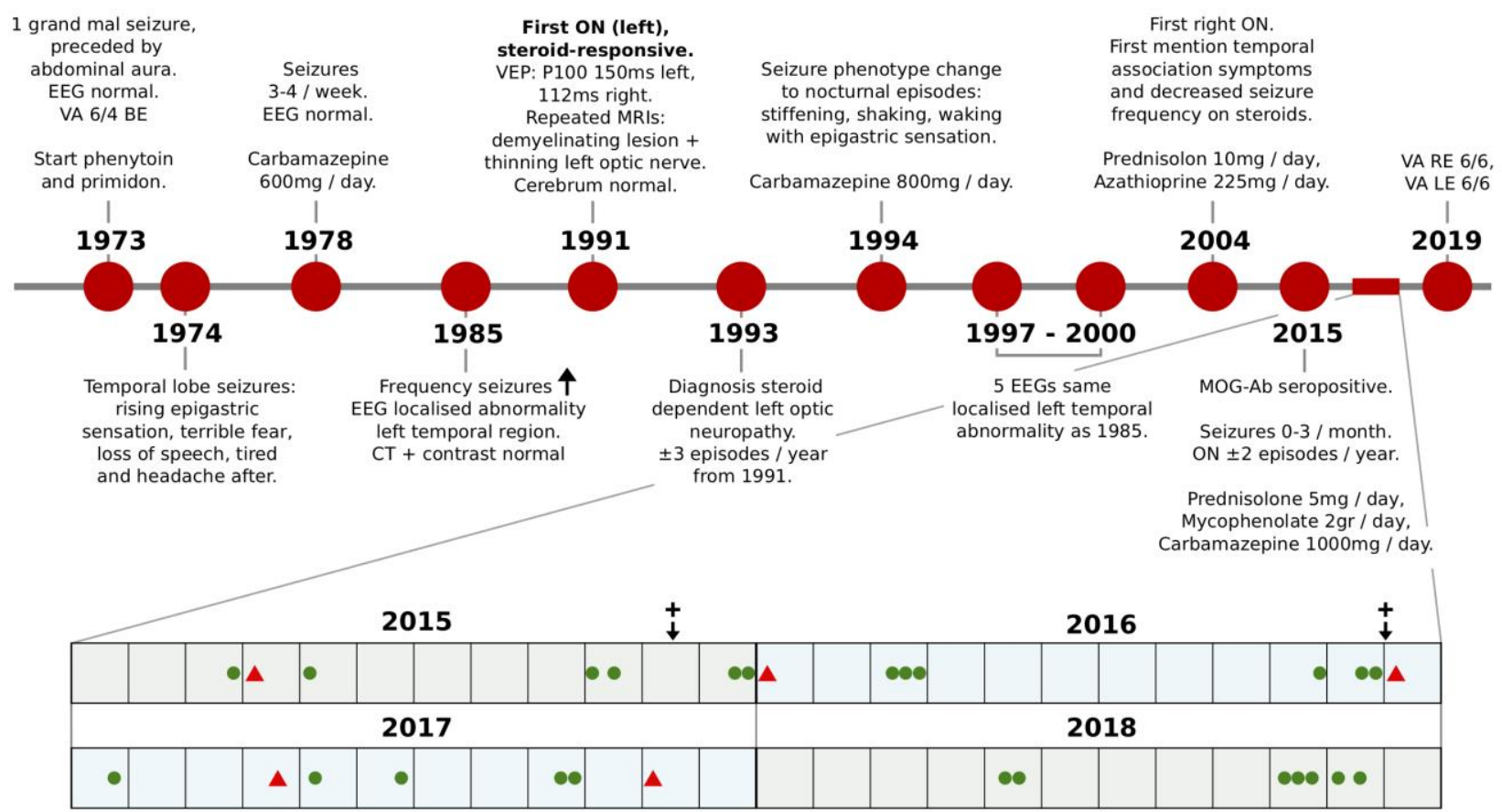

B

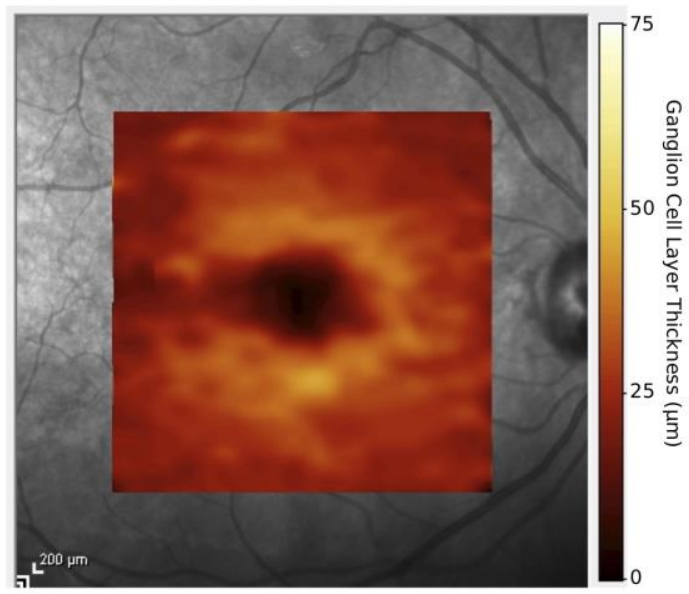

C

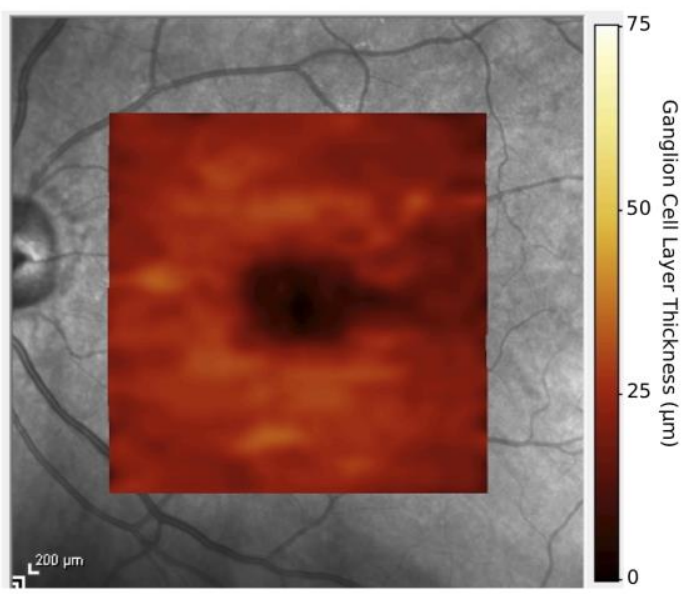


Figure 1 (A) Longterm follow-up of a MOG-Ab seropositive female patient with rrelapsing epilepsy and associated relapsing ON. Key symptoms, signs, test results and treatments are summarised. In 2015 our patient started her own diary (see inset) because she thought that her seizures (green dots) were synchronised in time to her attacks of relapsing ON (red triangles). Within this four year period, three out of five ON relapses were preceded by increased epilepsy activity by only 1-2 days. Both seizures and ON stopped on treatment with oral corticosteroids. On some occasions seizures re-occurred upon corticosteroid taper, as is the case with the seizures seen in October and November 2018. The timing of both positive MOG serum tests is noted with the arrow and + . The optical coherence tomography documented severe ganglion cell layer atrophy in (B) the right macula (volume $0.25 \mathrm{~mm}^{3}$ on $1,2.22,3.45 \mathrm{~mm}$ grid) and (C) more profoundly in the left macula (volume $0.20 \mathrm{~mm}^{3}$ ). This remained stable over the past six years. 


\section{References}

[1] Stellmann, J.-P.; Krumbholz, M.; Friede, T.; Gahlen, A.; Borisow, N.; Fischer, K.; Hellwig, K.; Pache, F.; Ruprecht, K.; Havla, J.; Kümpfel, T.; Aktas, O.; Hartung, H.-P.; Ringelstein, M.; Geis, C.; Kleinschnitz, C.; Berthele, A.; Hemmer, B.; Angstwurm, K.; Young, K. L.; Schuster, S.; Stangel, M.; Lauda, F.; Tumani, H.; Mayer, C.; Zeltner, L.; Ziemann, U.; Linker, R. A.; Schwab, M.; Marziniak, M.; Bergh, F. T.; Oy, U. H.-v.; Neuhaus, O.; Zettl, U.; Faiss, J.; Wildemann, B.; Paul, F.; Jarius, S.; Trebst, C. and Kleiter, I. (2017). Immunotherapies in neuromyelitis optica spectrum disorder: efficacy and predictors of response, Journal of Neurology, Neurosurgery \& Psychiatry 88 : 639-647.

[2] Petzold, A.; Woodhall, M.; Khaleeli, Z.; Tobin, W. O.; Pittock, S. J.; Weinshenker, B. G.; Vincent, A.; Waters, P. and Plant, G. T. (2019). Aquaporin-4 and myelin oligodendrocyte glycoprotein antibodies in immune-mediated optic neuritis at long-term follow-up., Journal of Neurology, Neurosurgery \& Psychiatry 90 : 1021-1026. https://doi.org/10.1136/jnnp$\underline{2019-320493}$

[3] Foiadelli, T.; Gastaldi, M.; Scaranzin, S.; Franciotta, D. and Savasta, S. (2020). Seizures and myelin oligodendrocyte glycoprotein (MOG) antibodies: Two paradigmatic cases and a review of the literature., Multiple Sclerosis and related disorders 41 : 102011. [4] Kidd, D.; Burton, B.; Plant, G. and Graham, E. (2003). Chronic relapsing inflammatory optic neuropathy (CRION), Brain 126 : 276-284.

[5] Plant, G. T.; Sibtain, N. A. and Thomas, D. (2011). Hyperacute corticosteroid treatment of optic neuritis at the onset of pain may prevent visual loss: a case series., Mult Scler Int $2011: 815068$. 